\title{
A Fast-Convergent Modulation Integral Observer for Online Detection of the Fundamental and Harmonics in Grid-Connected Power Electronics Systems
}

\author{
Boli Chen, Gilberto Pin, Wai Man Ng, Member, IEEE, Thomas Parisini, Fellow, IEEE, \\ and S.Y. Ron Hui, Fellow, IEEE
}

\begin{abstract}
Harmonics detection is a critical element of active power filters. A previous review has shown that the Recursive Discrete Fourier Transform and the Instantaneous p-q Theory are effective solutions to extracting power harmonics in single-phase and three-phase power systems, respectively. This paper presents the operating principle of a new modulation function integral observer algorithm that offers a fast solution for the extraction of the fundamental current and the total harmonic current when compared with existing methods. The proposed method can be applied to both single- and three-phase systems. The observerbased algorithm has an advantageous feature of being able to be tuned offline for a specific application, having fast convergence and producing estimated fundamental component with high circularity. It has been tested with both simulations and practical measurements for extracting the total harmonic current in a highly efficient manner. The results have confirmed that the proposed tool offers a new and highly effective alternative to the smart grid industry.
\end{abstract}

Index Terms - fundamental extraction, harmonics detection, active power filters

\section{INTRODUCTION}

$\mathrm{N}$ onlinear electric loads draw non-sinusoidal currents from the power supplies. Such harmonics have been identified as key factor for poor power quality and a range of adverse effects, including but not limited to, electromagnetic interference (EMI), overheating of cables, and low power factors [1]-[4]. Since the 1980's, the power electronics research community has responded with the solutions of active power

Manuscript received August 11 2015; accepted May 6 2016. This work was supported in part by the Hong Kong Research Council under the Theme-based Research Fund T23-701/14-N.

Boli Chen is with the Department of Electrical \& Electronic Engineering, Imperial College London (e-mail: boli.chen10@imperial.ac.uk).

G. Pin is with Electrolux, Italy (e-mail: gilbertopin @alice.it)

W.M. Ng is with the Department of Electrical \& Electronic Engineering, The University of Hong Kong (e-mail: wmng@eee.hku.hk).

T. Parisini is with the Department of Electrical \& Electronic Engineering, Imperial College London (e-mail: t.parisini@imperial.ac.uk).

S.Y.R. Hui is with Department of Electrical \& Electronic Engineering, The University of Hong Kong (e-mail: ronhui@eee.hku.hk) and also with Imperial College London (e-mail: r.hui@imperial.ac.uk). filters (APF) to deal with the power harmonics problems [5][10]. In a typical setup of an active filter as shown in Fig.1, one critical element is the real-time detection of the harmonic current. The control block involves a fast harmonic current detection so that a reference of the harmonic current can be derived for the power inverter to inject the harmonic current into the power line.

Despite such APF technologies have been commercialized [11], active research is still on-going in searching for effective real-time harmonics detection methods. In a recent survey [12], it is stated that "There exist many implementations supported by different theories (either in timeor frequency-domain), which continuously debate their performances proposing ever better solutions." The survey [12] provides a comprehensive comparison on six different methods covering (i) Discrete Fourier Transform (DFT), (ii) Recursive DFT, (iii) Synchronous Fundamental dq-Frame, (iv) $5^{\text {th }}$ Harmonic dq-Frame, (v) p-q Theory and (vi) $5^{\text {th }}$ Generalized Integrator. Methods (i), (ii) and (vi) are suitable for single-phase systems, and hence are suitable for three-phase ones as well. Methods (iii), (iv) and (v) can only be applied for three-phase systems and require filtering. In terms of settling time and phase error, the Recursive DFT and the p-q Theory methods offer better performance than the others in the comparison.

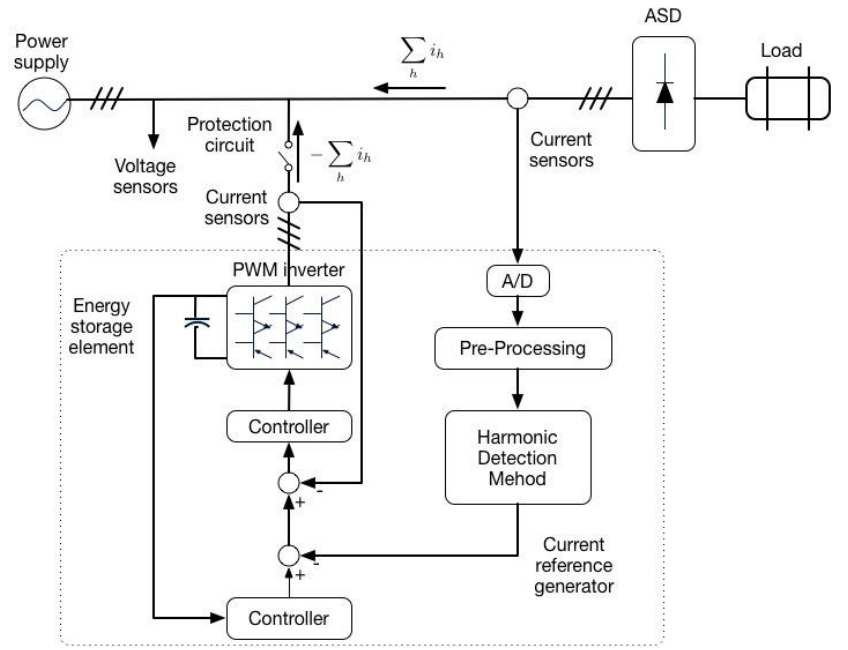

Fig.1 A typical setup of an Active Power Filter 
The methods described in [12] are familiar ones in the power electronics and power systems communities. However, significant progress has been achieved in signal processing and some of the emerging signal-processing methods are also applicable to harmonics detection. The frequency domain detection methods, such as discrete-time Fourier transform (DFT) and the sliding DFT [14]-[17] represent the most commonly used techniques for their fast convergence speed and higher accuracy comparing to the time-domain methods. Despite their successful applications, the existing methods based on DFT also suffer from two main drawbacks: (1) a synchronous sampling is needed, (2) problems in processing the inter-harmonics may occur. On the other hand, in the timedomain, methods like the Kalman filter, adaptive Notch filter (ANF), Phase-locked-loop (PLL) and second-order generalized integrators (SOGI) are proposed. These methods usually ensure enhanced noisy immunity at the expense of longer transient time than frequency-domain algorithms [18][25]. Moreover, the implementation of time-domain methods is more instrumental in the context of real-time harmonic compensation. In [26], PLL, recursive DFT (RDFT), and discrete Kalman filtering (DKF) from aforementioned two categories are compared analytically in terms of real-time implementation.

Very recently, a novel modulation-function integral observer (MF observer) has been developed for general single-input single-output linear systems. It offers practically instantaneous convergence without high gain injection and complexity [27]. The novel theory reported in [27] is supported by a rigorous and rather complex mathematical analysis. Thus, it may not be obvious to power electronics engineers without the relevant theoretical background. It is the aim of this paper to explore the use of this new modulation integral observer for extracting the fundamental current and the total harmonic current, the knowledge of the latter being required in active power filtering applications. The practical relevance of this MF observer with active power filter applications is explained in this paper for the first time. It is discovered that this new method, although unknown to the power electronics and power system communities, can perform fundamental current and total harmonic current extraction quickly even in presence of unknown dc offsets. The fast-convergent MF observer is implemented in non-selective form to achieve a low computational burden for on-line total harmonics extraction. As confirmed by the simulation and experimental results, this new method is favorably compared with some of the best harmonics extraction methods commonly used in the active power filtering applications.

\section{BRIEF SUMMARY OF FREQUENCY-DOMAIN AND TIME- DOMAIN HARMONIC DETECTION METHODS}

Although conducted 10 years ago, the review in [12] still provides some important insights into the operating principles and the algorithm structures of different harmonic detection methods. In this section, the schematics of the main methods are used to highlight the differences of the methodologies in terms of their algorithm structures. The understanding of such differences and the schematic structures of the harmonic detection algorithms will form a basis for comparing various approaches, including the fast-convergent integral observer under investigation in this paper.

\section{A. Frequency-Domain Discrete Fourier Transform and Recursive DFT}

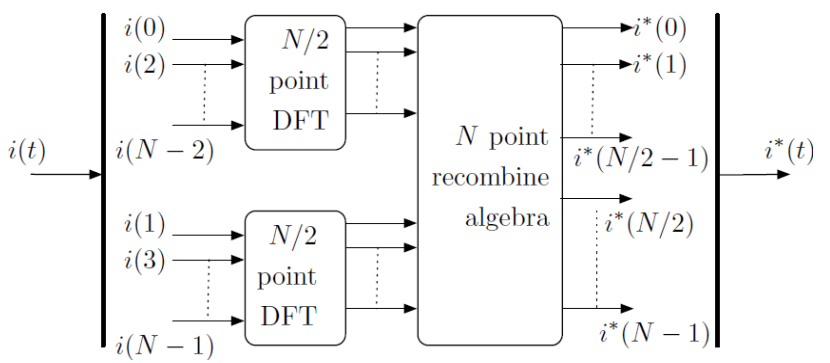

Fig.2 Schematic of the Discrete Fourier Transform

Fig.2 shows the schematic of the DFT method. The Recursive DFT (RDFT) is similar to the DFT, except that the samples are obtained within a moving window of the sampled data. Note that the DFT method and its variants require the measurements of the current only. There is no need to measure the mains voltage. Its ability to handle 1-phase measurements naturally indicates that it can be applied to a 3 -phase system. There are two ways to work out the total harmonic current.

- The first one is to obtain the sum of the dc component and fundamental component. By subtracting this sum from the input current waveform, the total harmonic current function can be obtained.

- The second way is to add several of the low-order current harmonic components together to form an approximate harmonic current.

\section{B. Time-Domain dq-Frame methods}

Unlike the DFT method, the time-domain dq-Frame approach requires the measurements of both of the mains voltages and currents for the 3 phases. Therefore, this method requires more sensors and is not suitable for single-phase applications. The phase currents are measured and transformed into the direct (d) and quadrature (q) components. The time-domain dq-Frame method can also be used in two ways as described for the DFT method.

(i) Synchronous fundamental dq-Frame method

Fig.3 shows the schematic of this method for extracting the harmonic components. The rotating frame of this method rotates at the fundamental frequency. This makes the fundamental component appearing as the dc component after the transformation in the rotating frame. With the use of a high-pass filter with a typical bandwidth of $25 \mathrm{~Hz}$ to $120 \mathrm{~Hz}$, the fundamental $\mathrm{d}$ - and q- current components can be eliminated. The high-pass-filter enables the d- and qcomponents of the harmonic currents $\left(\mathrm{i}_{\mathrm{d}} \sim\right.$ and $\left.\mathrm{i}_{\mathrm{q}} \sim\right)$ to go through. By doing a dq-abc transformation, the harmonic currents of the three phases can be re-constructed as $i_{a}{ }^{*}, i_{b}{ }^{*}$ and $i_{c} *$ in Fig.3. 


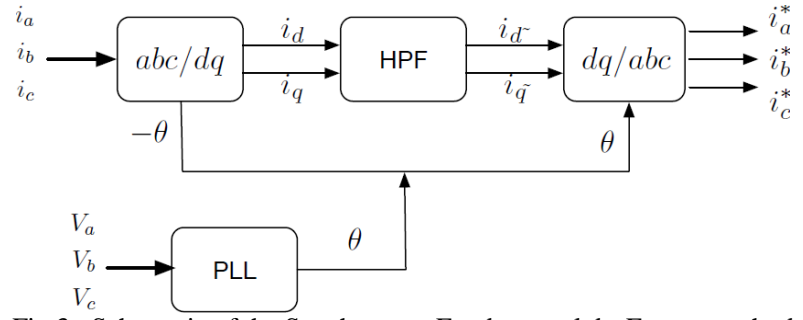

Fig.3 Schematic of the Synchronous Fundamental dq-Frame method

(ii) Synchronous harmonic dq-Frame method

The schematic of the harmonic dq-Frame is shown in Fig.4. Again, the three phase currents and voltages have to be measured. The procedures for extracting the harmonic currents are similar to that of the fundamental dc frame method. The major difference is that the rotating frames for the harmonics will be according to their respectively harmonic frequencies. Once the harmonic currents are obtained, they can be added together to form the total harmonic current. In practice, only several of the low-order harmonic currents are usually used to reconstruct a good approximation of the total harmonic currents for the three phases.

It is important to note that the Fundamental dq-Frame and Harmonic dq-Frame methods require the use of highpass or low-pass filters in the process of deriving the required current components. Consequently, such approach unavoidably introduces phase error, as previously discussed in [12].

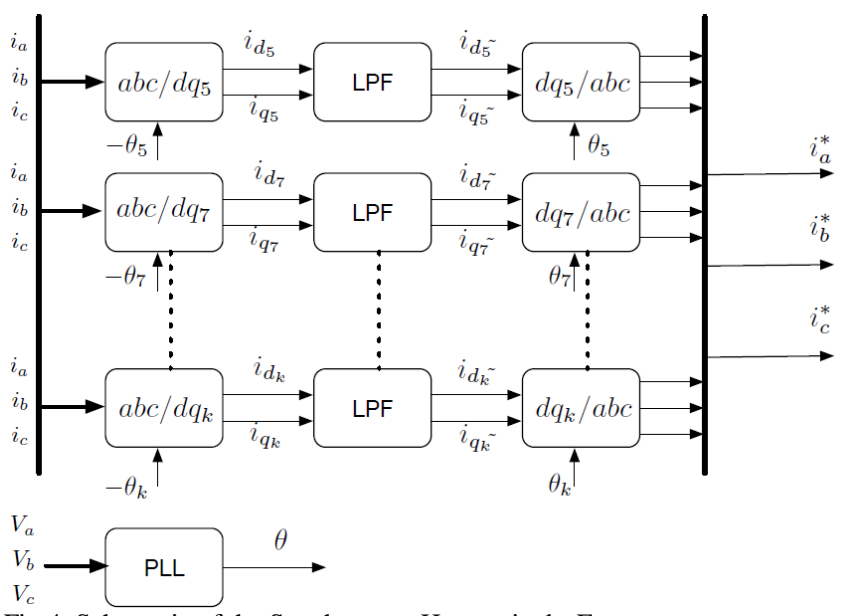

Fig.4 Schematic of the Synchronous Harmonic dq-Frame

(iii) Instantaneous p-q power theory

Another common approach for harmonic extraction is based on the instantaneous p-q power theory pioneered by Prof. Akagi [28]. The schematic is shown in Fig.5. Like the dq-Frame methods, this approach needs the measurements of the phase currents and voltages. Thus, it is suitable for 3phase systems, but not for single-phase ones. The phase voltage and current measurements are transformed into their respectively $\alpha$ and $\beta$ components. Then the real power $(p)$ and reactive power $(q)$ are obtained. With the use of high-pass filters, the harmonic real power $(\tilde{p})$ and harmonic reactive power $(\tilde{q})$ are obtained. The $\alpha$ and $\beta$ harmonic current components $\left(i_{\alpha}^{*}\right.$ and $\left.i_{\beta}^{*}\right)$ are then derived, and are eventually used to reconstruct the total harmonic currents for the three phases.

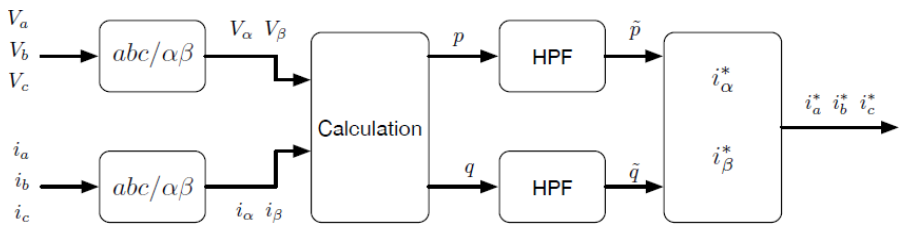

Fig.5 Schematic of the method based on the Instantaneous p-q Power Theory

In the next section, the proposed fast fundamental and harmonics estimator will be illustrated.

\section{MOdUlation Function INTEGRAL OBSERVER METHOD}

In In this section, a third-order MF observer is introduced for harmonic current extraction. The schematic of this method is shown in Fig.6. With the help of a modulation function that can be selected and tuned offline for a specific application, the line current samples are fed to the integral observer in order to obtain the sum of the dc component and the fundamental component of the current. The total harmonic current can then be derived by subtracting this sum from the line current. It should be noted that this method can be applied to a singlephase system. Therefore, it can be applied to 3-phase ones too.

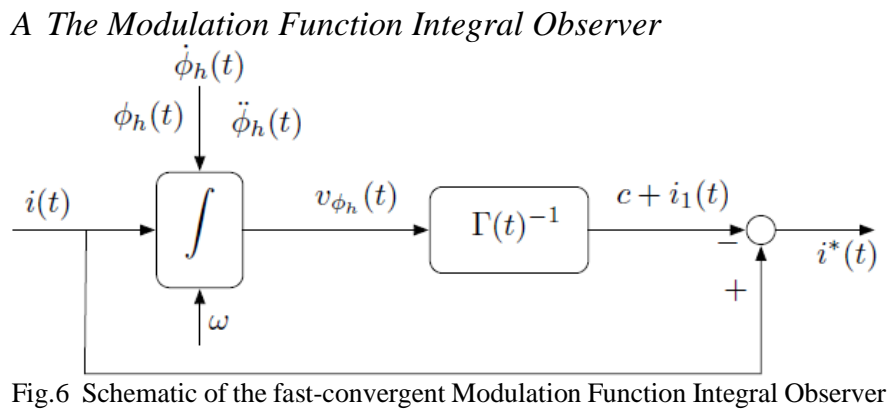

The line current $i(t)$ is expressed as:

$$
i(t)=c+i_{1}(t)+\sum_{k=2}^{N} i_{k}(t)
$$

where $c$ is the dc current component, $t$ is the time variable, $i_{1}(t)$ is the fundamental current, $i_{k}(t)$ is the $k^{\text {th }}$ current harmonic component and $\sum_{k=2}^{N} i_{k}(t)$ is the total harmonic current.

Let the fundamental current be:

$$
i_{1}(t)=a \sin (\omega t+\varphi)
$$

and

$$
y(t)=c+i_{1}(t)
$$

where $A$ is the amplitude of the fundamental current, $\omega$ is the angular frequency and $\varphi$ is the phase shift. It is important to note that the integral observer considers the total harmonic current as 'noise' initially. If $y(t)$ can be found by the observer, from (1) and (3), the total harmonic current can be obtained easily "by subtraction" as follows: 


$$
\sum_{k=2}^{N} i_{k}(t)=i(t)-y(t)
$$

Therefore, $y(t)$ is chosen to be the single output of the integral observer introduced in the following lines. Differentiating (3) twice leads to:

$$
\ddot{y}(t)=-\omega^{2} i_{1}(t)
$$

where $\ddot{y}(t)$ is the second-order time-derivative of $y(t)$. Based on (3) and (5), one can form the following expression:

$$
\left[\begin{array}{l}
y(t) \\
\ddot{y}(t)
\end{array}\right]=\left[\begin{array}{cc}
1 & 1 \\
0 & -\omega^{2}
\end{array}\right]\left[\begin{array}{c}
c \\
i_{1}(t)
\end{array}\right]
$$

Now, assume that the single output $y(t)$ is generated by a general $3^{\text {rd }}$ order state-space observable system,

$$
\left\{\begin{array}{l}
\dot{\mathbf{z}}(t)=\mathbf{A}_{Z} \mathbf{z}(t) \\
y(t)=\mathbf{c}_{Z}^{T} \mathbf{z}(t)
\end{array}\right.
$$

where we let

$$
\begin{aligned}
& \mathbf{z}(t)=\left[\begin{array}{l}
z_{1}(t) \\
z_{2}(t) \\
z_{3}(t)
\end{array}\right] \\
& =\left[\begin{array}{c}
c+a \sin (\omega t+\varphi) \\
\omega \cos (\omega t+\varphi) \\
\omega^{2} c
\end{array}\right]
\end{aligned}
$$

and

$$
\mathbf{A}_{z}=\left[\begin{array}{ccc}
0 & 1 & 0 \\
-\omega^{2} & 0 & 1 \\
0 & 0 & 0
\end{array}\right], \quad \mathbf{c}_{Z}=\left[\begin{array}{l}
1 \\
0 \\
0
\end{array}\right]
$$

It can be easily seen that

$$
\begin{gathered}
z_{1}(t)=y(t) \\
z_{3}(t)=\ddot{y}(t)+\omega^{2} y(t)
\end{gathered}
$$

Clearly, the total harmonic current can be derived as long as $z_{1}(t)$ is available. However, in the following lines, we will show that the dc offset and the fundamental signal can be individually recovered by using the state vector $\mathbf{z}(t)$. Since only $z_{1}(t)$ and $z_{3}(t)$ are relevant in the calculation, the following matrix equations can be derived:

$$
\mathbf{z}_{\mathbf{e}}(t)=\mathbf{A y}(t)
$$

where

$$
\begin{gathered}
\mathbf{z}_{\mathbf{e}}(t)=\left[\begin{array}{l}
z_{1}(t) \\
z_{3}(t)
\end{array}\right], \mathbf{A}=\left[\begin{array}{ll}
1 & 0 \\
\omega^{2} & 1
\end{array}\right] \text { and } \\
\mathbf{y}(t)=\left[\begin{array}{c}
y(t) \\
\ddot{y}(t)
\end{array}\right]
\end{gathered}
$$

thus leading to

$$
\mathbf{y}(t)=\mathbf{A}^{-1} \mathbf{z}_{\mathbf{e}}(t)
$$

The $2 \times 1$ state column vector $\mathbf{z}_{e}(t)$ can be related to the $3 \times 1$ state column vector $\mathbf{Z}(t)$ as:

$$
\mathbf{z}_{\mathbf{e}}(t)=\mathbf{I}_{\mathbf{e}} \mathbf{z}(t)
$$

with

$$
\mathbf{I}_{\mathbf{e}}=\left[\begin{array}{lll}
1 & 0 & 0 \\
0 & 0 & 1
\end{array}\right]
$$

In view of (13), (12) and (6), the fundamental and the dc component modelled in the right hand side of (6) can be retrieved by simple algebra (see A.3-A.5 in Appendix I).

\section{$B$ The Modulation Function and the Modulation Matrix}

The modulation integral observer uses a set of the timemonomial modulating functions (see [27]). In the case addressed in this paper, the specific functions take on the form

$$
\phi_{h}(t)=\frac{w_{h} t^{2 n-h}}{(2 n-h) !} \quad \text { for } \quad h \in\{1,2, \ldots, n\}
$$

where $n$ is the order of the system (i.e., $n=3$ for the $3^{\text {rd }}$ order observer in this case) and $w_{h}>0$ is a suitable positive weighting factor. According to [27], the chosen modulation functions must satisfy:

$$
\frac{d^{n} \phi_{h}(0)}{d t}=0
$$

The reason for selecting the modulation function (14) that is characterized by condition (15) is provided in the Appendix II. With the modulation function selected, the modulation function matrix is defined as:

$$
\Gamma(t)=\left[\begin{array}{lll}
\ddot{\phi}_{1}(t) & -\dot{\phi}_{1}(t) & \phi_{1}(t) \\
\ddot{\phi}_{2}(t) & -\dot{\phi}_{2}(t) & \phi_{2}(t) \\
\ddot{\phi}_{3}(t) & -\dot{\phi}_{3}(t) & \phi_{3}(t)
\end{array}\right]
$$

where $\dot{\phi}_{h}(t)$ and $\ddot{\phi}_{h}(t)$ are the first and second derivatives of $\phi_{h}(t)$ for $h \in\{1,2,3\}$. For any choice of $w_{h}$, it holds that

$$
\begin{aligned}
& \phi_{1}(t) \neq \phi_{2}(t) \neq \phi_{3}(t), \forall t>0 \\
& \dot{\phi}_{1}(t) \neq \dot{\phi}_{2}(t) \neq \dot{\phi}_{3}(t), \forall t>0 \\
& \ddot{\phi}_{1}(t) \neq \ddot{\phi}_{2}(t) \neq \ddot{\phi}_{3}(t), \forall t>0
\end{aligned}
$$

thus $\Gamma(t)$ is invertible for any $t>0$.

\section{Remarks:}

Although $\phi_{h}(t), h=1,2,3$ are monotonically increasing functions with respect to $t$, they are only computed over $0 \leq$ $t \leq T_{\Delta}+T_{r}$ due to the action of reset (which is described later on), thus evading the issue of data overflow. In this respect, excessively large $w_{h}$ is not advisable. The same rule is applicable to $\dot{\phi}_{h}(t)$ and $\ddot{\phi}_{h}(t)$. Moreover, by observing the pattern of $\boldsymbol{\Gamma}(t), w_{h}, h=1,2,3$ should be chosen such that the resultant $\phi_{h}(t), h=1,2,3$ are enough separated to facilitate the computation of matrix inverse). It is also important to note that the elements in the matrix of (17) can be pre-determined in an offline manner in the interval: $0 \leq t \leq T_{\Delta}+T_{r}$. Therefore, the matrix inversion is done only once. These predetermined values can be stored in memory for real-time implementation.

With the availability of the modulation function matrix, the following matrix equation can be formed: 


$$
\left[\begin{array}{c}
v_{\phi 1}(t) \\
v_{\phi 2}(t) \\
v_{\phi 3}(t)
\end{array}\right]=\left[\begin{array}{lll}
\ddot{\phi}_{1}(t) & -\dot{\phi}_{1}(t) & \phi_{1}(t) \\
\ddot{\phi}_{2}(t) & -\dot{\phi}_{2}(t) & \phi_{2}(t) \\
\ddot{\phi}_{3}(t) & -\dot{\phi}_{3}(t) & \phi_{3}(t)
\end{array}\right]\left[\begin{array}{c}
z_{1}(t) \\
z_{2}(t) \\
z_{3}(t)
\end{array}\right]
$$

Hence,

$$
\begin{aligned}
& {\left[\begin{array}{l}
z_{1}(t) \\
z_{2}(t) \\
z_{3}(t)
\end{array}\right]=\left[\begin{array}{lll}
\ddot{\phi}_{1}(t) & -\dot{\phi}_{1}(t) & \phi_{1}(t) \\
\ddot{\phi}_{2}(t) & -\dot{\phi}_{2}(t) & \phi_{2}(t) \\
\ddot{\phi}_{3}(t) & -\dot{\phi}_{3}(t) & \phi_{3}(t)
\end{array}\right]^{-1}\left[\begin{array}{c}
v_{\phi 1}(t) \\
v_{\phi 2}(t) \\
v_{\phi 3}(t)
\end{array}\right]} \\
& \mathbf{z}(t)=\boldsymbol{\Gamma}(t)^{-1} \mathbf{v}_{\boldsymbol{\varphi}}(t)
\end{aligned}
$$

where the elements of column vector $\mathbf{v}_{\varphi}(t)=\left[\begin{array}{lll}v_{\phi 1}(t) & v_{\phi 2}(t) & v_{\phi 3}(t)\end{array}\right]^{T}$ can be obtained from the practical measurements of the line current $i(t)$. It follows that

$$
v_{\varphi h}(t)=\int_{0}^{t}\left[\dddot{\phi}_{h}(\tau) i(\tau)+\omega^{2} \dot{\phi}(\tau) i(\tau)\right] d \tau
$$

Thus,

$$
\dot{v}_{\varphi h}(t)=\left[\dddot{\phi}_{h}(t)+\omega^{2} \dot{\phi}_{h}(t)\right] \cdot i(t)
$$

By observation (19), in order to avoid error-accumulation due to the integration in case the measurement of $i(t)$ is noisy, we adopt a discrete-time deflation strategy which is described in the following lines. Let us define the time variable

$$
t_{k}=T+(k+1) T_{r}
$$

where $T_{\Delta}$ is the initial time, $T_{r}$ is a repetitive period (note: within each repetitive period are many small sampling periods), and $k$ is a natural number, the first derivative of $\dot{v}_{\phi h}(t)$ can be obtained from the practical measurement of the line current $i(t)$. When $k=0, t=t_{0}=T_{\Delta}+T_{r}$, this forms the first repetitive period. Beyond this time, the time variable lies within the next repetitive period $T_{r}$ (i.e. $t_{k}<t<t_{k+1}$ ).

$$
\dot{v}_{\phi h}(t)= \begin{cases}{\left[\dddot{\phi}(t)+\omega^{2} \dot{\phi}(t)\right] \cdot i(t)} & \text { for } 0 \leq t<t_{0} \\ {\left[\dddot{\phi}\left(t-t_{k}+T_{\Delta}\right)+\omega^{2} \dot{\phi}\left(t-t_{k}+T_{\Delta}\right)\right] \cdot i(t)} & \text { for } t_{k}<t<t_{k+1}\end{cases}
$$

The time integrals of $\dot{v}_{\phi h}(t)$ in (21) will lead to the values of $v_{\phi h}(t)$ for $h=\{1,2,3\}$. At the alternation of each repetitive period, the values of $v_{\phi h}(t)$ should be reset as:

$$
v_{\phi h}\left(t_{k}^{+}\right)=\mathbf{E}_{h}^{T} \boldsymbol{\Gamma}\left(T_{\Delta}\right) \boldsymbol{\Gamma}^{-1}\left(T_{\Delta}+T_{r}\right) \mathbf{v}_{\varphi}\left(t_{k}\right) \quad \text { for } t=t_{k}
$$

where $\mathbf{E}_{1}=\left[\begin{array}{l}1 \\ 0 \\ 0\end{array}\right], \quad \mathbf{E}_{2}=\left[\begin{array}{l}0 \\ 1 \\ 0\end{array}\right]$ and $\mathbf{E}_{3}=\left[\begin{array}{l}0 \\ 0 \\ 1\end{array}\right]$.

Note that the line current is sampled at a relatively high speed within each repetitive period, and the integral values of (21) can provide the time-domain vector $\mathbf{v}_{\varphi}(t)$ continuously at each sampling instant, then the state vector $\mathbf{z}(t)$ can be obtained from (17).

$$
\mathbf{z}(t)= \begin{cases}\boldsymbol{\Gamma}^{-1}(t) \cdot \mathbf{v}_{\varphi}(t) & \text { for } T_{\delta} \leq t \leq t_{0} \\ \boldsymbol{\Gamma}^{-1}\left(t-t_{k}+t_{\Delta}\right) \cdot \mathbf{v}_{\varphi}(t) & \text { for } t_{k} \leq t \leq t_{k+1}\end{cases}
$$

where $T_{\delta}$ is a small time. It is needed because the modulation function matrix is not invertible at $t=0$. Thanks to (9), $z_{1}(t)$ represents an estimate of $y(t)$, then the total harmonic current can be obtained from (4). In the practical implementation, the proposed integral observer algorithms can be discretized as shown in the Appendix I.

\section{Simulation Study OF CONVERGENT RATES AND TRACKING ERRORS}

In order to evaluate the performance of the methods under consideration, a simulation study based on the conditions similar to those set in [12] has been conducted. The characteristic of the line currents are tabulated in Table I and such reference current waveform is shown in Fig.7. We compare the MF observer with two well-known methods that are successfully used in the active power filter context: the recursive DFT, $\mathrm{p}-\mathrm{q}$ theory, and a variant of the adaptive notch filtering (ANF) [24] recently proposed for this typical application. The realizable algorithms of each method are described in the Appendix. For the MF observer, the weighting factors are tuned offline to be $w_{1}=1, w_{2}=2$ and $w_{3}=3 . T_{r}=T_{\Delta}$ $=0.1$. For the Instantaneous p-q power theory, the high-pass filter has a cut off frequency of $200 \mathrm{rad} / \mathrm{s}$. A pair of RDFT filters are employed with order 0 and 1 for the offset and fundamental respectively. The ANF is tuned with $\zeta_{a}=\zeta_{b}=\zeta_{c}=$ 0.8 and $\zeta=30$.

\section{TABLE I CHARACTERISTICS OF LINE CURRENT}

\begin{tabular}{|c|c|c|}
\hline Indices & Amplitude & Phase \\
\hline Offset & 3 & 0 \\
\hline Fundamental & 30 & $0,120,240$ \\
\hline $5^{\text {th }}$ harmonic & 10 & $5 \times(0,120,240)$ \\
\hline $7^{\text {th }}, 11^{\text {th }}, 13^{\text {th }}, 17^{\text {th }}$ & 1.5 & $h \times(0,120,240)$ \\
\hline & & \\
\hline
\end{tabular}

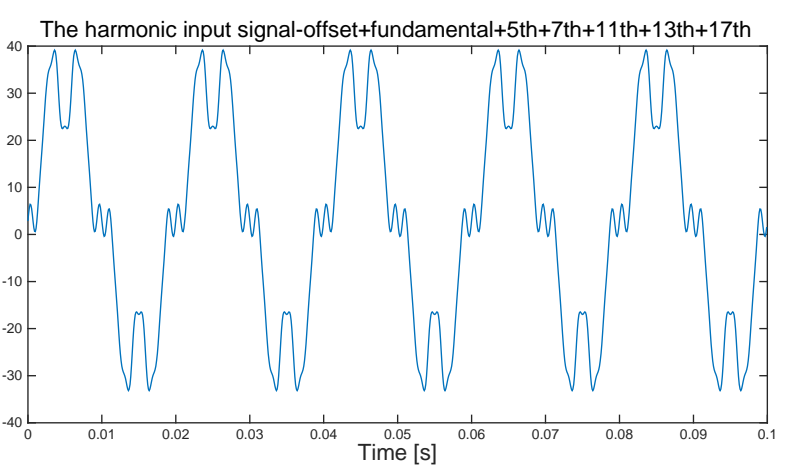

Fig.7 A reference current waveform constructed from the components tabulated in Table I the for the simulation study 


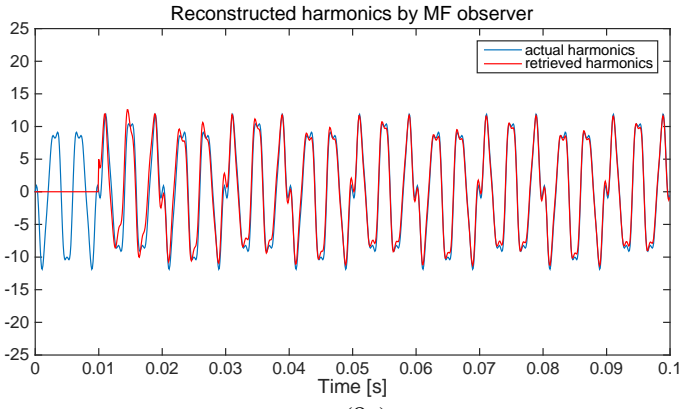

(8a)

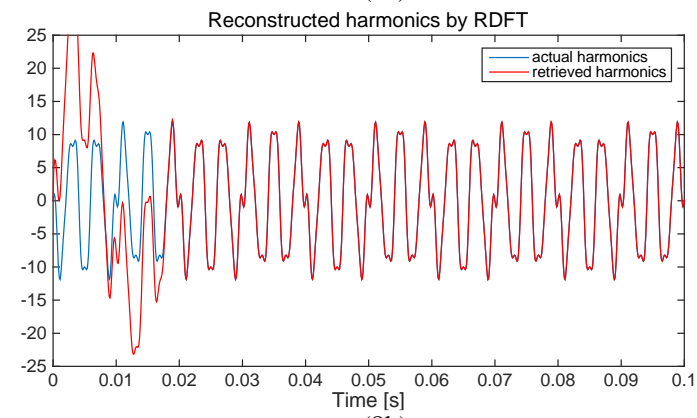

(8b)

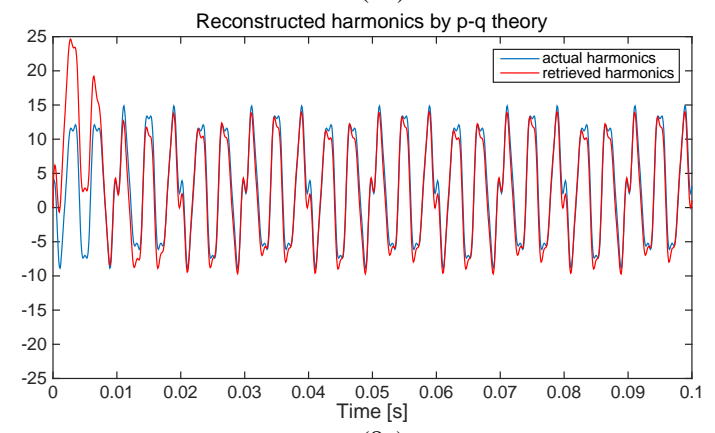

(8c)

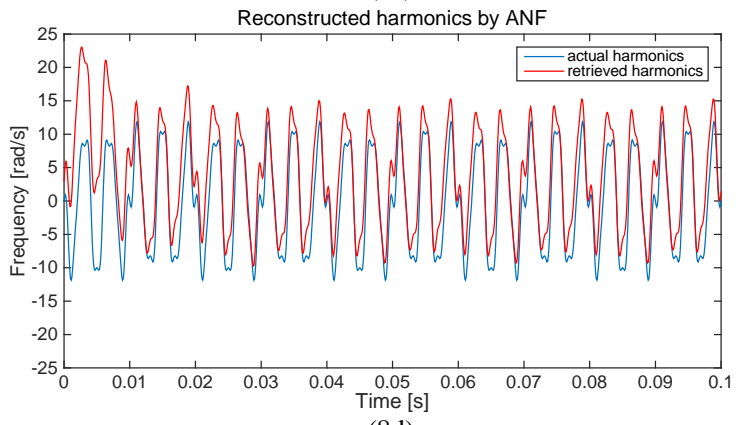

(8d)

Fig.8 Comparison of the actual harmonics and the extracted harmonics based on the (a) MF observer, (b) RDFT, (c) Instantaneous p-q power theory and (d) the ANF methods.

Figs. 8(a)-(d) show the actual harmonic current and the extracted current waveform in the time domain based on the (i) MF observer, (ii) RDFT, (iii) Instantaneous p-q power theory and (iv) the ANF methods. For the sake of comparison, the tracking errors of these four methods are also examined by subtracting the actual harmonic waveform from the extracted waveforms. These tracking errors are compared in Fig.9, where the steady-state errors of the ANF method (which gives the largest error among the four methods) are used as the error tolerances (as indicated by the two long dotted lines of \pm 7 in Fig.9), the convergence times of these methods to reach the tolerance region are tabulated in Table II. The $\mathrm{p}-\mathrm{q}$ theory method and the MF method have the fastest convergence performance.

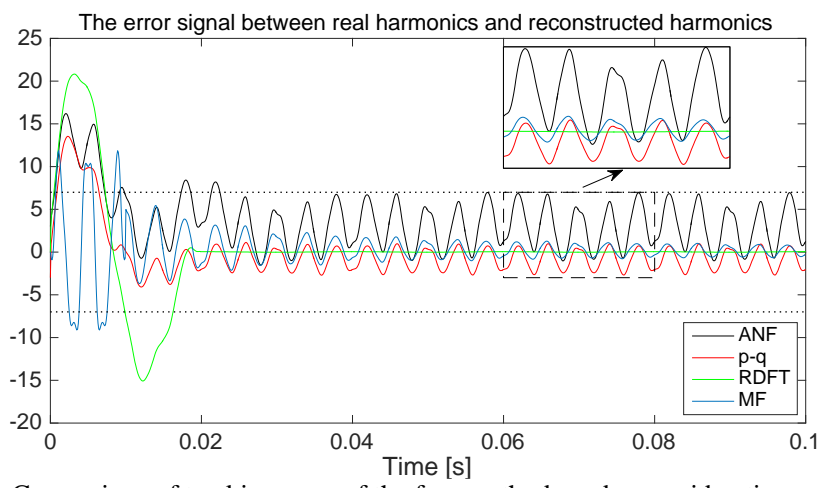

Fig.9 Comparison of tracking error of the four methods under consideration

TABLE II CONVERGENCE TIMES OF THE FOUR METHODS

\begin{tabular}{|c|c|c|c|c|}
\hline & MF & RDFT & p-q & ANF \\
\hline Convergence time & $10 \mathrm{~ms}$ & $20 \mathrm{~ms}$ & $<10 \mathrm{~ms}$ & $>20 \mathrm{~ms}$ \\
\hline
\end{tabular}

The MF observer takes about $10 \mathrm{~ms}$ to track the harmonic waveform and is faster than RDFT and ANF, which require at least $20 \mathrm{~ms}$. Although RDFT offers minimal steady-state error in a steady-state situation, it is important to note that fast convergence offers advantage to signal detection applications in which fast transients and frequency variations are common. Based on these simulation results, a comparative table with respect to the steady state error is also constructed as shown in Table III. The following points can be noted:

1. The results shown in the Table II confirm that the MF performs the best among all the approaches

2. RDFT is very smooth in steady state; therefore the relative estimation error decreases as more cycles are considered.

3. ANF has the worst steady state behavior; therefore the relative error increases as more cycles are considered.

TABLE III COMPARISON OF RMS VALUES OF THE ESTIMATION ERRORS ON FIG.9

\begin{tabular}{|l|l|l|c|c|}
\hline & MF & RDFT & p-q & ANF \\
\hline $0-0.02$ s (10 cycles) & $1.0-$ pu & 2.17 -p.u. & 1.09 -p.u. & 1.48 -p.u. \\
\hline $0-0.1$ s (50 cycles) & $1.0-$ pu & 2.05 -p.u. & $1.13-$ p.u. & $2.40-$ p.u. \\
\hline
\end{tabular}

\section{EXPERIMENTAL VERIFICATION}

A third-order MF observer has been implemented to derive the fundamental and the dc bias current only. The total harmonic current is then obtained by subtracting dc and fundamental current components from the line current. Based on the platform suggested in [12], the total harmonic currents, settling times, phase errors and overshoots of the following methods are compared with the practical measurements.

- MF observer

- Recursive DFT,

- The Instantaneous p-q Theory method

- $\quad$ Adaptive Notch Filter (ANF) method [24] 
The algorithms of the $3^{\text {rd }}$ order MF observer are implemented in a discretized form which is included in Appendix I. The algorithms of the RDFT and ANF methods also can be referred to the Appendix I. The algorithms of the instantaneous p-q theory are well known and simply follow the block diagrams as shown in Fig.5. The algorithms under consideration are implemented in a dSpace DS1006 system. Fig.10 shows the hardware setup, which includes a programmable power supply (AMETEK CSW11100), three inductors of slightly different inductance values, and three resistive loads. The line currents of the three phase system are displayed in Fig. 11.

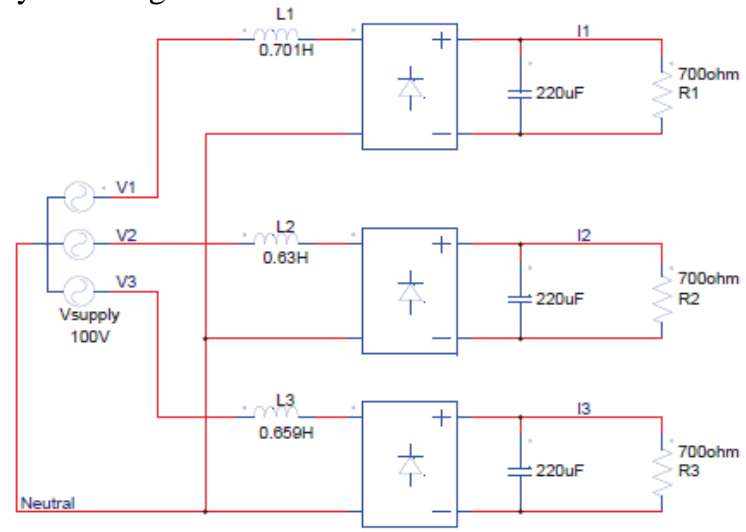

Fig.10 Schematic of the three phase converter with resistive loads.

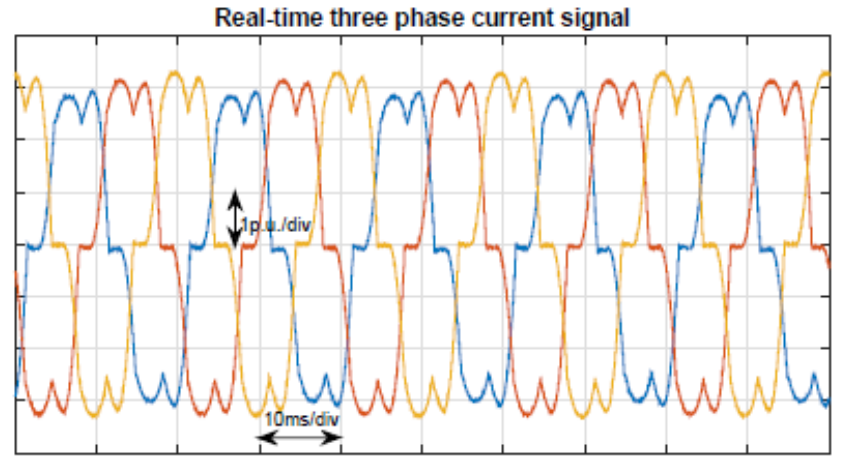

Fig.11 Practical line currents of the three-phase system with a converter-resistor load.

\section{A Extraction of the fundamental current component}

Since the Instantaneous p-q power theory method uses a high-pass filter to remove the fundamental component in order to derive the total harmonic current (Fig.5), it is not included in this comparison because it does not generate the fundamental current. Only the experimental results of the MF observer, RDFT and ANF methods are included in this section. Under a fixed sampling frequency of $10 \mathrm{kHz}$, the same dSpace system is used to calculate the fundamental current in real time. The analogue of this estimated fundamental current is outputted to a digital storage oscilloscope (DSO-X 3034A). The estimated fundamental currents of the MF observer, RDFT and ANF methods are captured and displayed in Figs. 12(a), 12(b) and 12(c), respectively.

In order to observe the deviation of these waveforms from a pure sinusoidal waveform, let us transform the estimated sinewave into a unit "circle" by picking arbitrarily consecutive 200 samples in one cycle. It is worth noting that a perfect timedomain sinusoidal function can be represented as an ideal circle on the 2-D plane. By comparing the trajectory of the measured current with a perfect circuit, any deviation from the perfect circle can be observed. One method of measuring the quality of the extracted fundamental component is the concept of Circularity [29], which defines the formula for Circularity $(C)$

$$
C=\frac{4 \pi A}{P^{2}}
$$

with $A$ and $P$ the area and the perimeter enclosed by of the circular figure, respectively. Meanwhile, the isoperimetric theorem indicates that $C=1$ only holds for an ideal circle. To this end, the circularity of each figure is evaluated in the following way:

1. Divide the shape into circular sectors with radius $r_{i}, i=$ $1,2, \cdots, 200$ based on the samples.

2. Approximation of the area $A=\sum_{i=1}^{200} \frac{1}{200} \pi r_{i}^{2}$

3. The perimeter $P$ of the curve is approximated by the sum of the distances between every two adjacent samples.

As such, the vector trajectories of one period of three estimated fundamental current waveforms are plotted and compared with a perfect circle in Figs. 13(a), 13(b) and 13(c). It can be observed that the MF observer method generate the best fundamental current with the least amount of noise and distortion. For the sake of completeness, the circularity values of the MF observer, the RDFT and the ANF have been obtained and are recorded in Table IV. It can be seen that the curve generated by MF has the circularity closest to 1 (i.e with the least absolute deviation from 1.0).

TABle IV COMPARISON OF CIRCUlarity OF THE EXTRACTED FUNDAMENTAL COMPONENTS

\begin{tabular}{|c|c|c|c|}
\hline & $\begin{array}{c}\text { Circularity } \\
C\end{array}$ & $\begin{array}{c}\text { Absolute deviation } \\
|C-1.0|\end{array}$ & Figure \\
\hline MF observer & 1.0017 & 0.0017 & $13(\mathrm{a})$ \\
\hline RDFT & 0.8925 & 0.1075 & $13(\mathrm{~b})$ \\
\hline ANF & 0.8883 & 0.1117 & $13(\mathrm{c})$ \\
\hline
\end{tabular}

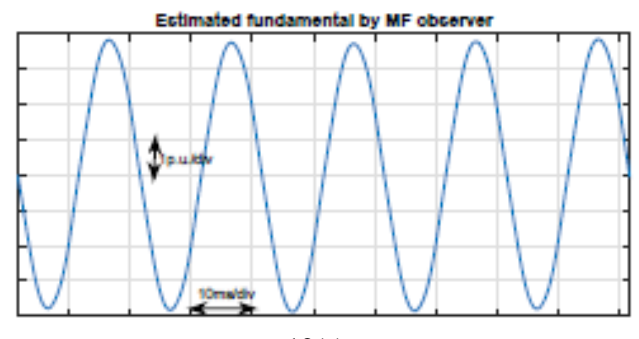

12(a)

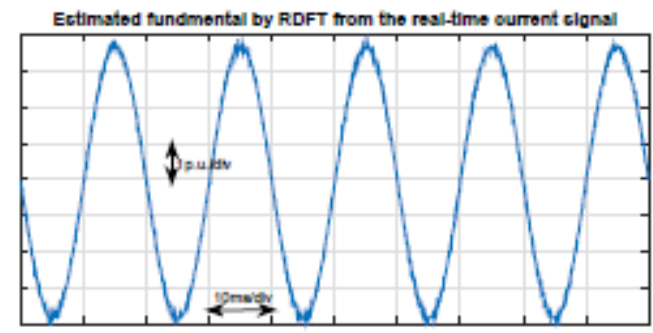

12(b) 


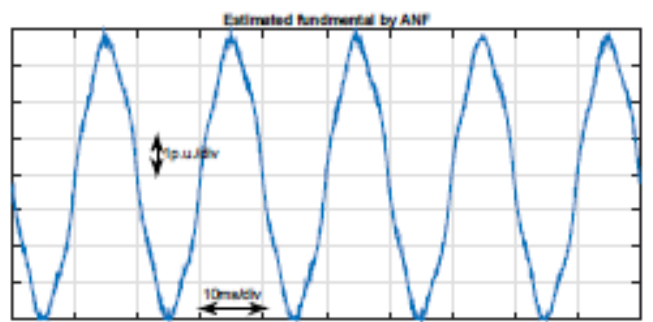

12(c)

Fig.12 The estimated fundamental currents obtained from the (a) MF observer, (b) RDFT and (c) ANF methods.

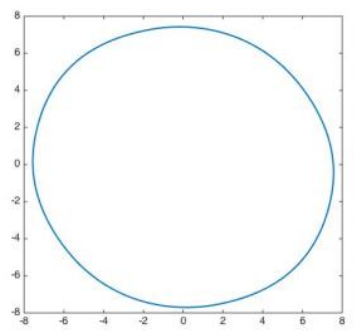

13(a)

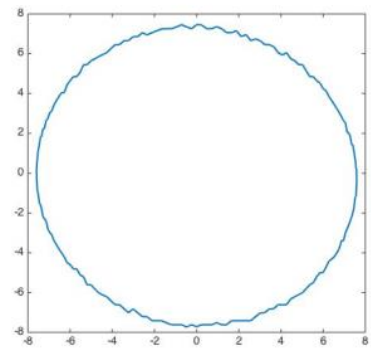

13(b)

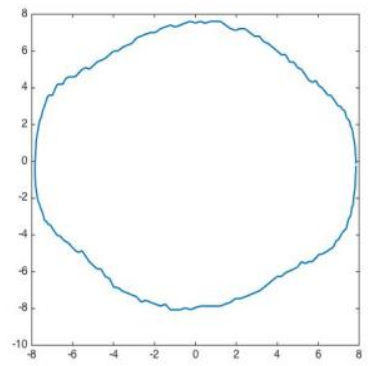

13(c)

Fig.13 Comparison of the trajectories of the fundamental currents estimated by the (a) MF observer, (b) RDFT and (c) ANF methods with the perfect sinusoidal trajectory.

\section{$B$ Detection of the total harmonic current}

The second set of evaluation involves all of the four methods under consideration. This time, the total harmonic currents estimated by the four methods are captured. Their harmonic spectra are then plotted and compared with the harmonic spectrum of the mains current, which is included in Fig.14 as a reference. Figs. 15(a), 15(b), 15 (c) and 15(d) show the frequency spectra of the estimated total harmonic currents obtained from the (a) MF observer, (b) RDFT, (c) Instantaneous p-q power Theory and (d) the ANF methods, respectively. Comparing with the spectrum of the measured line current in Fig.14 and ignoring the part of the spectrum up to about $200 \mathrm{~Hz}$ (to remove the effect of the fundamental current), the spectra of MF observer, RDFT method and the ANF method are close to that of the line current.

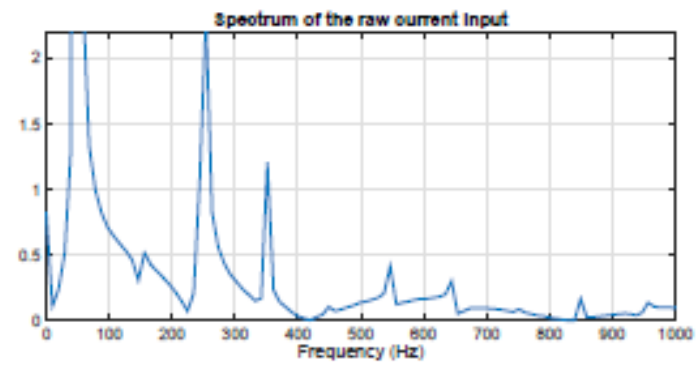

Fig.14 The measured frequency spectrum of measured line current of Fig.11
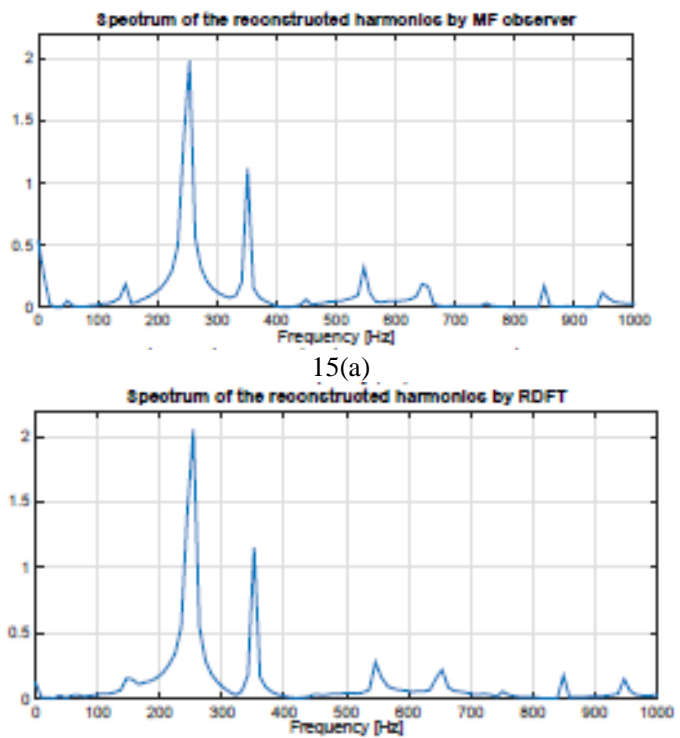

15 (b)

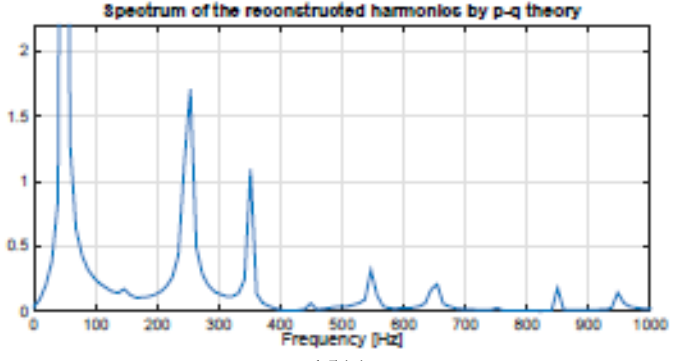

15(c)

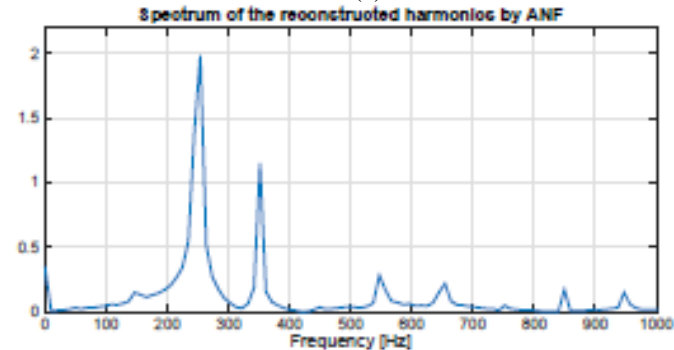

$15(\mathrm{~d})$

Fig.15 Measured harmonic spectra of (a) the MF observer, (b) the RDFT, (c) the Instantaneous p-q power theory and (d) the ANF methods.

\section{Overall Comparison}

With the results obtained, the performance of the four methods can be summarized as tabulated in Table V. The RDFT has generally good performance. The only limitation is its 
requirement for synchronous sampling that may lead to accuracy issues when the frequency is changing dynamically.

The Instantaneous p-q power theory requires the measurements of voltage and current. It is a fast method to generator the total harmonic current. But the introduction of filter in the process of harmonic extraction inevitably introduces phase error. The proposed MF observer has fast convergence for tracking the total harmonic current. Among the three methods that can extract the fundamental current, the MF observer offers the highest quality in terms of circularity. Because of the high quality of the fundamental current, its resultant total harmonic current (which is obtained by subtracting the fundamental current from the line current) is also of high quality.

\section{CONCLUSIONS}

This paper presents the first practical implementation of a new modulation function integral observer for the extraction of the fundamental current and the total harmonic current of a three-phase nonlinear load. The observer algorithm has been adopted specifically for fundamental current and total harmonic current extraction. It has been explained step by step so that power electronics researchers and engineers can implement it without understanding the full details of the highly mathematical observer theory given in [27] in a more general setting. Based on the concept of circularity, it has been practically confirmed that this method has excellent performance in obtaining a high-quality representation of the fundamental current in real time from a highly nonlinear current waveform. Consequently, the total harmonic current, which is obtained by subtracting the fundamental current from the total line current, is also of high quality. Such high-quality signal extraction capability has been favorably compared with the RDFT, the Instantaneous p-q power theory (that are commonly used in the power electronics community) and the ANF methods. The fast convergent feature of this observer has also been demonstrated in a simulation study. Based on the comparison provided, it is concluded that the modulation function integral observer method offers a fast and practical online solution to obtaining the fundamental component and the total harmonic content of a highly nonlinear signal. Such functions can find many power electronics applications (including active power filters) in which highly nonlinear currents are involved.

TABLE V COMPARISON OF FOUR METHODS FOR HARMONICS EXTRACTION

\begin{tabular}{|c|c|c|c|c|}
\hline & RDFT & p-q Theory & MF observer & ANF \\
\hline Required measurements & I only & $\mathrm{V}$ and $\mathrm{I}$ & I only & I only \\
\hline Response time & $20 \mathrm{~ms}$ & $10 \mathrm{~ms}$ & $10 \mathrm{~ms}$ & $30 \mathrm{~ms}$ \\
\hline Steady state accuracy & accurate & phase error & Accurate & less accuracy \\
\hline Selective harmonic compensation & yes & no & potential yes $^{1}$ & yes \\
\hline 1-ph/3-ph applications & single/three & three & single/three & single/three \\
\hline Need for synchronous sampling & yes & no & no & No \\
\hline Computational load $(\mathrm{p}-\mathrm{u})^{2}$ & 0.5-p.u. & 2-p.u. & 1-p.u. & 1.3-p.u. \\
\hline Estimation of the dc offset & yes & no & yes & no \\
\hline Circularity of fundamental & Fair & Not Applicable & Excellent & Fair \\
\hline
\end{tabular}

\section{APPENDIX I}

1. MF observer

Discretized algorithm of (21)-(23) by a trapezoidal integration rule (see A1 at the bottom of this page):

Hence,

$$
\mathrm{z}(\mathrm{k})=\left\{\begin{array}{c}
0,0 \leq k \leq N_{\epsilon} \\
(\boldsymbol{\Gamma}(k))^{-1} \mathbf{v}(k), N_{\in} \leq k \leq N_{0} \\
\left(\boldsymbol{\Gamma}\left(k-N_{k}+N_{\Delta}\right)\right)^{-1} \mathbf{v}(k), N_{k} \leq k \leq N_{k+1}
\end{array}\right.
$$

\footnotetext{
${ }^{1}$ Multiple harmonics can be dealt with by a higher order MF observer (e.g. $2 n+1$-th order for $n$ harmonics and the dc offset), which omitted here for sake of unnecessary complexity
}

where $T_{s}$ is the fixed sampling period, $N_{k}=\frac{t_{k}}{T_{s}}, k \in \mathbb{N}, N_{\Delta}=$ $\frac{T_{\Delta}}{T_{S}}, N_{r}=\frac{T_{r}}{T_{S}}, N_{\in}=\frac{T_{\epsilon}}{T_{S}}$.

Particularly, in the discrete-time case $T_{r}, T_{\Delta}$ and $T_{\in}$ are chosen as multiples of the sampling time Ts.

Finally

$$
\begin{array}{r}
\mathbf{z}_{e}(\mathrm{k})=\mathbf{I}_{e} \mathbf{z}(\mathrm{k}) \\
\mathbf{y}(\mathrm{k})=\mathbf{A}^{-1} \mathbf{z}_{e}(k) \\
{\left[\begin{array}{c}
c \\
i_{1}(k)
\end{array}\right]=\left[\begin{array}{cc}
1 & 1 \\
0 & -\omega^{2}
\end{array}\right]^{-1} \mathbf{y}(\mathrm{k})}
\end{array}
$$

\section{RDFT}

\footnotetext{
${ }^{2}$ The computation time is evaluated by Matlab under a fixed simulation example
} 
It has been show that the RDFT is very convenient in extracting a specific harmonic component from a given current $i(t)$ by the following recursive algorithm $(j$ is the order of the desired harmonic

$$
h_{j}(k)=\frac{2}{N}(i(k)-i(k-N))+W^{j} h_{j}(-1)
$$

which is driven by its equivalent transfer function $G_{j}(z)$ between the input $i(z)$ and the extracted harmonic signal $h_{j}(z)$, :

$$
G_{j}(z)=\frac{h_{j}(z)}{i(z)}=\frac{1}{N} \frac{1-z^{-N}}{1-W^{j} z^{-1}}
$$

3. $\mathrm{p}-\mathrm{q}$ theory

The reader is referred to Figure 5 for the overall scheme.

4. Adaptive notch filtering (ANF)

Herein, a recent ANF [24] is picked for the sake of comparison and is reviewed in the following equations (the single-phase structure):

$$
\left\{\begin{array}{c}
\ddot{h}_{j}(t)=-j^{2} \theta(t)^{2} h_{j}(t)+2 \zeta_{j} \theta(t) e(t) \\
e(t)=i(t)-\sum_{l=1}^{n} \dot{h}_{l} \\
\dot{\theta}(t)=-\gamma \theta(t) h_{1}(t) e(t)
\end{array}\right.
$$

where $i(t)$ represents the current signal from each phase respectively, $\theta$ is the estimate of the fundamental frequency $\omega, j=1,2, \cdots, n$ is the order of the selected harmonic, $\zeta_{j}, \gamma$ are all positive adjustable parameters balancing the converging speed and accuracy, $l$ is the sequence of the harmonic that is estimated. The continuous-time algorithm can simply discretized by Euler or trapezoidal rules for digital implementation.

\section{APPENDIX II}

A nominal state observer (i.e., Luenberger Observer) is applicable for the linear system (7). However, only asymptotic convergence is guaranteed (i.e., the estimated state $\hat{\mathrm{z}}(\mathrm{t}) \rightarrow$ $z(t)$, when $t \rightarrow \infty)$, because of the initial error driven by the unknown initial condition. In order to circumvent this restriction and to propose an observer that can converge within an arbitrary small finite time, we annihilate the influence of the unknown initial condition by exploiting an integral operator (see (18)) with respect to the designed modulation function. The next Lemma introduced in [27] is instrumental for the following description.

Lemma 1 (Modulated signal's derivative): Consider a signal $x(t), t \geq 0$ that admits a $i$-th order derivative, and a $i$-th order differentiable modulation function $V_{\phi} x(t), t \geq 0$. It holds that:

$$
\begin{aligned}
{\left[V_{\phi} x^{(i)}\right](t)=\sum_{j=0}^{i-1} } & (-1)^{i-j-1} x^{(j)}(t) \phi^{(i-j-1)}(t) \\
& +\sum_{j=0}^{i-1}(-1)^{i-j} x^{(j)}(0) \phi^{(i-j-1)}(0) \\
& +(-1)^{i}\left[V_{\phi^{i}} x\right](t)
\end{aligned}
$$

where we let the $\frac{d^{i}}{d t^{i}} x(t) \triangleq x^{(i)}(t)$ for the sake of brevity and we define the modulation operator by

$$
\left[V_{\phi} x\right](t) \triangleq \int_{0}^{t} \phi(\tau) x(\tau) d \tau .
$$

The above Lemma motivates us to eliminate the unknown initial conditions $x(0), \frac{d^{1}}{d t^{1}} x(0), \frac{d^{2}}{d t^{2}} x(0), \cdots$ by choosing a modulation function $\phi(t)$, such that

$$
\frac{d^{n}}{d t^{n}} \phi(0)=0
$$

Thanks to (A9), we design the following modulating function

$$
\phi_{h}=\frac{w_{h} t^{2 n-h}}{(2 n-h) !}, \quad h=\{1,2, \cdots n\}
$$

$$
\left\{\begin{array}{c}
v_{\phi_{h}}(k+1)=v_{\phi_{h}}(k)+\frac{1}{2} T_{s}\left(\left(\dddot{\phi}_{h}(k+1)+\omega^{2} \dot{\phi_{h}}(k+1)\right) i(k+1)+\left(\dddot{\phi}_{h}(k)+\omega^{2} \dot{\phi_{h}}(k)\right) i(k)\right), 0 \leq k<N_{0} \\
v_{\phi_{h}}(k+1)=v_{\phi_{h}}(k)+\frac{1}{2} T_{s}\left(\begin{array}{c}
\left(\dddot{\phi}_{h}\left(k+1-N_{k}+N_{\Delta}\right)+\omega^{2} \dot{\phi_{h}}\left(k+1-N_{k}+N_{\Delta}\right)\right) i(k+1)+ \\
\left(\dddot{\phi}_{h}\left(k-N_{k}+N_{\Delta}\right)+\omega^{2} \dot{\phi}_{h}\left(k-N_{k}+N_{\Delta}\right)\right) i(k)
\end{array}\right), N_{k}<k<N_{k+1} \\
v_{\phi_{h}}\left(N_{k}^{+}\right)=E_{h}^{T} \boldsymbol{\Gamma}\left(N_{\Delta}\right) \boldsymbol{\Gamma}\left(N_{\mathrm{r}}+N_{\Delta}\right)^{-1} \mathbf{v}\left(N_{k}\right), k=N_{k} .
\end{array}\right.
$$


where the index $h$ allows a set of modulation functions in this form distinguished by different parameter $w_{h}$ and order $h$. Admittedly, the choice of the modulation function satisfying (A9) is not unique. For this application paper, we only show this typical function as a simple example. Although the modulation function plays an important role in this methodology, the selection does not vary from application to application; the modulation functions satisfying (A9) are applicable for the state estimation problem of any linear system in which the harmonic model can be embedded.

Acknowledgement:

This work was partially supported by the HK Research Grant Council under the Theme-based Research Fund T23-701/14-N.

\section{REFERENCES}

[1] A. Segura and P. Sanchez, "Experimental measurement of noncharacteristic harmonic power generated by thyristor pulse-controlled $\mathrm{ac} / \mathrm{dc}$ three phase converters," in Proceedings of the IEEE International Symposium on Industrial Electronics, 1996, pp. 549-554.

[2] A. Phadke and J. Harlow, "Generation of abnormal harmonics in highvoltage ac-dc power systems," IEEE Transactions on Power Apparatus and Systems, vol. 87, no. 3, pp. 873-883, 1968.

[3] K. Patil and W. Gandhare, "Effects of harmonics in distribution systems on temperature rise and life of XLPE power cables," in International Conference on Power and Energy Systems (ICPS), 2011, pp. 1-6.

[4] J. D. H. Harold A. Gauper and A. McQuarrie, "Generation of abnormal harmonics in high-voltage ac-dc power systems," IEEE Spectrum, vol. 8 , no. 10 , pp. $32-43,1971$.

[5] M. Lowenstein, "Improving power factor in the presence of harmonics using low voltage tuned filters," in Conference Record of the 1990 IEEE Industry Applications Society Annual Meeting, 1990, pp. 1767-1773.

[6] H. Akagi, "New trends in active filters for power conditioning," IEEE Transactions on Industry Applications, vol. 32, no. 6, pp.1312-1322, 1996.

[7] A. C. Bhim Singh, K. Al-Haddad and, "A review of active filters for power quality improvement," IEEE Transactions on Industrial Electronics, vol. 46, no. 5, pp. 960-971, 1999.

[8] H. Akagi, "Active harmonic filters," in Proc. of the IEEE, vol. 93, no. 12, 2005, pp. 2128-2141.

[9] H. Akagi, "Trends in active power line conditioners," IEEE Transactions on Power Electronics, vol. 9, no. 3, pp. 263-268, 1994.

[10] F. Z. Peng, "Application issues of active power filters," IEEE Industry Applications Magazine, vol. 4, no. 5, pp. 21-30, 1998.

[11] "Advanced Activer Power Filters: A flexible and adaptive solution for central or de-central harmonic mitigation", [Online]. Available: http://www.danfoss.com/NR/rdonlyres/87CF6FEA-CA08-4518-AE368534104DFA38/0/VLTAdvancedActiveFilterFactsheetMP012A023.pd $\mathrm{f}$

[12] L. Asiminoaei, F. Blaabjerg, and S. Hansen, "Evaluation of harmonic detection methods for active power filter applications," in Applied Power Electronics Conference and Exposition, 2005. APEC 2005. Twentieth Annual IEEE, 2005, pp. 635-641.

[13] D. Kucherenko and P. Safronov, "A comparison of time domain harmonic detection methods for compensating currents of shunt active power filter," in IEEE International Conference on Intelligent Energy and Power Systems (IEPS), no. 12, 2014, pp. 40-45.

[14] B. McGrath, D. Holmes, and J. Galloway, "Power converter line synchronization using a discrete fourier transform (dft) based on a variable sample rate," IEEE Trans. on Power Electron., vol. 20, no. 4, pp. 877-884, 2005.

[15] S. Gonzalez, R. Garcia-Retegui, and M. Benedetti, "Harmonic computation technique suitable for active power filters," IEEE Trans. on Ind. Electron., vol. 54, no. 5, pp. 2791-2796, 2007.

[16] E. Jacobsen and R. Lyons, "The sliding DFT," IEEE Signal Process. Mag., vol. 20, no. 2, pp. 74-80, 2003.

[17] E. Jacobsen and R. Lyons, "An update to the sliding DFT," IEEE Signal Process. Mag., vol. 21, no. 1, pp. 110-111, 2004.
[18] A. A. Girgis, W. B. Chang, and E. B. Makram, "A digital recursive measurement scheme for on-line tracking of power system harmonics," IEEE Trans. on Power Delivery, vol. 6, no. 3, pp. 1153-1160, 1991.

[19] X. Yuan, W. Merk, H. Stemmler, and J. Allmeling, "Stationary-frame generalized integrators for current control of active power filters with zero steady-state error for current harmonics of concern under unbalanced and distorted operating conditions," IEEE Trans. Ind. Appl., vol. 38, no. 2, pp. 523-532, 2002.

[20] P. Rodriguez, A. Luna, I. Candela, R. Mujal, R. Teodorescu, and F. Blaabjerg, "Multiresonant frequency-locked loop for grid synchronization of power converters under distorted grid conditions," IEEE Trans. Ind. Electron., vol. 58, no. 1, pp. 127-138, 2011.

[21] Y. F. Wang and Y. W. Li, "Three-phase cascaded delayed signal cancellation pll for fast selective harmonic detection," IEEE Trans. on Ind. Electron., vol. 60, no. 4, pp. 1452-1463, 2013.

[22] M. Karimi-Ghartemani and M. R. Iravani, "Measurement of harmonics/inter-harmonics of time-varying frequencies," IEEE Trans. on Power Delivery, vol. 20, no. 1, pp. 23-31, 2005.

[23] M. Mojiri, M. Karimi-Ghartemani, and A. Bakhshai, "Time-domain signal analysis using adaptive notch filter," IEEE Trans. on Signal Processing, vol. 55, no. 1, pp. 85-93, 2007.

[24] D. Yazdani, A. Bakhshai, G. Joos, and M. Mojiri, "A real-time threephase selective-harmonic-extraction approach for grid-connected converters," IEEE Transactions on Industrial Electronics, vol. 56, no. 10, pp. 4097-4106, 2009.

[25] M. Mojiri, M. Karimi-Ghartemani, and A. Bakhshai, "Processing of harmonics and inter-harmonics using an adaptive notch filter," IEEE Trans. on Power Delivery, vol. 25, no. 2, pp. 534-542, 2010.

[26] V. M. Moreno, M. Liserre, A. Pigazo, and A. D. Aquila, "A comparative analysis of real-time algorithms for power signal decomposition in multiple synchronous reference frames," IEEE Trans. on Power Electron., vol. 22, no. 4, pp. 1280-1289, 2007.

[27] G. Pin, B. Chen, and T. Parisini, "The modulation integral observer for linear continuous-time systems," in Proc. of the IEEE European Control Conference, Linz, 2015.

[28] H. Kim and H. Akagi, "The instantaneous power theory based on mapping matrices in three-phase four-wire systems," in IEEE Proc. of Power Conversion Conference, 1997, pp. 361-366.

[29] V. Blasjo, "The Evolution of the Isoperimetric Problem," Amer. Math. Monthly 112: 526-566. 